\title{
ANÁLisis CUANTITATIVO DE LOS ELEMENTOS ALEANTES PRINCIPALES PRECIPITADOS EN BILLETS DE ALUMINIO DE ALEACIÓN 6063 EN EQUIPO DE FUNDICIÓN DE COLADA CONTINUA HORIZONTAL PARA EL PROCESO DE EXTRUSIÓN
}

\author{
QUANTITATIVE ANALYSIS OF MAIN \\ PRECIPITATE ALLOYING ELEMENTS IN \\ ALUMINUM ALLOY 6063 CAST BILLETS MADE \\ IN HORIZONTAL CONTINUOUS CASTING \\ MACHINE FOR THE EXTRUSION PROCESS
}

\author{
Hernán Alberto Navas-Moscoso ${ }^{1, *}$, Wilson Henry Vaca-Ortega ${ }^{2}$, Juan Gilberto
}

Paredes-Salinas $^{2}$, Fabián Rodrigo Morales-Fiallos ${ }^{2}$, Diego Fernando Núñez-Núñez ${ }^{2}$

\section{Resumen}

La escasez de estudios in situ sobre procesos de colada continua horizontal en aleaciones de aluminio, impulsa la necesidad de investigar la influencia de temperatura, velocidad y materia prima en billets de aluminio homogenizados AA6063. Aplicando ensayos de espectrometría, permiten cuantificar el porcentaje en peso de aleantes principales magnesio y silicio. Estos elementos al precipitar forman siliciuro de magnesio $\left(\mathrm{Mg}_{2} \mathrm{Si}\right)$ durante el proceso de homogenizado. Para este estudio se produjo billets de aleación normal y experimental, demostrando una relación inversa entre los niveles de siliciuro de magnesio y dureza Brinell; así también la influencia de bajos niveles de porcentaje en peso de $\mathrm{Mg}_{2} \mathrm{Si}$ en la reducción de la resistencia a la tracción en el material.

Palabras clave: aleación 6063, billet, precipitado.

\section{Abstract}

Lack of in situ studies about horizontal continuous casting process applied in production of aluminum alloy 6063 cast billets, promotes to investigate the direct influence of: temperature, velocity and raw material. Applying spectrometric tests to quantify the weight percent of main alloying elements such as Magnesium and Silicon. These elements precipitate to form magnesium silicide $\left(\mathrm{Mg}_{2} \mathrm{Si}\right)$ during homogenization process. For this study it was necessary to produce billets with normal and experimental alloy levels to demonstrate an inverse relation between magnesium silicide and Brinell hardness. Equally important, the effect of reduction of the material tensile strength due to low levels of magnesium silicide.

Keywords: Alloy 6063, billet, precipitate.

\footnotetext{
$\overline{1, *}$ Facultad de Ingeniería Civil y Mecánica - Universidad Técnica de Ambato, Ambato - Ecuador. Autor para correspondencia hernannavasmoscoso@gmail.com

${ }^{2}$ Facultad de Ingeniería Civil y Mecánica, Universidad Técnica de Ambato, Ambato - Ecuador.

Recibido: 21-11-2016, aprobado tras revisión: 20-12-2016

Forma sugerida de citación: Navas, H.; Vaca, W.; Paredes, J.; Morales, F.; Núñez, D. (2017). «Análisis cuantitativo de los elementos aleantes principales precipitados en billets de aluminio de aleación 6063 en equipo de fundición de colada continua horizontal para el proceso de extrusión». INGENIUS. N. ${ }^{\circ} 17$, (Enero-Junio). pp. 42-50. ISSN: 1390-650X.
} 


\section{Introducción}

El proceso de fundición de colada continua horizontal con equipo tipo HERTWICH es aplicable para convertir chatarra de aluminio en billets o lingotes de aleación según sea su clase, sin embargo, cabe recalcar que HERTWICH diseñó este tipo de equipo para el proceso de remelting. [1]

Pocas empresas poseen la capacidad para incluir en su proceso de fundición este tipo de equipo, sea esto debido a la complejidad y singularidad del mismo, ya que se requiere de meticulosos procedimientos manejados de forma confidencial en cada empresa; no obstante, la formación del billet de aluminio de manera horizontal representa un gran reto, por lo cual es necesario un control continuo de los principales elementos que constituyen la aleación en cada turno de producción. [2] Sin embargo, uno de los métodos no destructivos que ayuda a proporcionar información con tolerancia más estrecha, rápida y predilecto para monitorear los niveles de aleación 6063 es el espectrómetro de emisión óptica porque tiene la ventaja de ser un ensayo que permite en tiempo real conocer si la aleación cumple con estándares nacionales e internacionales. [2]

Varios estudios han permitido obtener a partir de modelos matemáticos, ecuaciones que son aplicadas en muchos estudios experimentales con resultados favorables que confirman un alto nivel de exactitud y confianza en los resultados.

La distribución longitudinal de los elementos aleantes principales (silicio y magnesio) demuestran la naturaleza del proceso de colada continua horizontal, así también la importancia del proceso de homogenizado como complemento para la formación de una solución homogénea; el resultado será un material con características mecánicas intrínsecas de la aleación 6063 para que al final del proceso de extrusión los perfiles que sean sometidos a tratamiento térmico T5 o T6 adquieran las propiedades ideales cumpliendo los estándares de calidad.

\subsection{Aleación 6063}

La aleación 6063 pertenece a la serie 6000 de aleaciones de aluminio. Estas poseen como elementos aleantes principales al magnesio y silicio porque ambos contribuyen a la formación de siliciuro de magnesio, este compuesto ayuda a fortalecer su estructura cristalina durante el proceso de solución sólida. [3, 4]

Forma parte de una de las cuatro series de aleaciones que acepta tratamiento térmico, sin embargo, la aleación 6063 al estar incluida en la serie 6000 admite el temple y la maduración natural o artificial. [3,4]

\subsection{Propiedades del magnesio en aleación}

Las aleaciones de la serie 6000 se benefician de la alta solubilidad en sólido del magnesio, incrementando su resistencia a la corrosión, ductilidad y conductividad térmica. También mejoran su respuesta al proceso de anodizado. [2] [4]

Está demostrado que el magnesio incrementa la ductilidad y fortalecimiento de la aleación; sin embargo, al sobrepasar una concentración de 0,7 en porcentaje de peso, disminuye el límite de solubilidad sólida y dificulta la extrusión del billet. [2] [4]

\subsection{Propiedades del silicio en aleación}

El silicio en las aleaciones de la serie 6000 es un elemento endurecedor, debido a su alta solubilidad en aluminio es agregado en colada en pequeños trozos o barras para mezclarse con otros elementos en el aluminio fundido sin llegar a sobrecalentar el metal. [2] [4]

Facilita la respuesta de los perfiles extruidos al proceso de envejecido artificial para proveer de un mayor fortalecimiento que aquel por medio de envejecido natural. [2] [4]

Garantiza una óptima fluidez de la aleación y consigue un adecuado moldeo; también reduce el coeficiente de expansión térmica del material. [4]

\subsection{El siliciuro de magnesio}

El siliciuro de magnesio en la aleación 6063 produce un efecto de fortalecimiento por medio de la solución sólida molecular fijando las dislocaciones. [3] Como referencia a señalar en la serie 6000 , indica la dificultad de considerar dentro de un diagrama ternario por separado al silicio y magnesio con el aluminio; por lo cual se encuentra descrito en memorias de varias investigaciones que el $\mathrm{Mg}_{2} \mathrm{Si}$ forma un sistema cuasibinario. [3] Estudios experimentales han determinado en aleaciones industriales 6063 que precisan un exceso de silicio para controlar niveles de magnesio y para dar lugar a la formación de siliciuro de magnesio el cien por ciento de magnesio se precipita. [2] [4]

\subsection{Proceso de colada continua horizontal}

Es un proceso diseñado para empresas extrusoras de aluminio con el fin que puedan realizar el proceso de refundido con chatarra de alta calidad proveniente de su propio proceso de extrusión. [1]

Este equipo sirve para una operación continua logrando maximizar la producción en periodos de tiempo desde un mínimo de tres días y reportados en mejor de los casos tres semanas ininterrumpidas. [1]

Por medio de la técnica de molde abierto se logra producir con un mismo equipo: lingotes, billets (cilindros), barras T, entre otros; cada uno de ellos con diferentes dimensiones y longitudes con la integración de un sistema con sierra de corte. En la Figura 1 se aprecia las partes principales del equipo de colada continua horizontal, además a continuación se establece brevemente una descripción de los mismos: 
Equipo de carga: Compuesto por la máquina de carga de material la cual posee en su plataforma de trabajo una báscula para pesar los elementos que se van a adicionar en el horno de fusión.

Horno de fusión: Posee doble cámara siendo la primera cámara de fusión (melting) y la segunda cámara de colado (casting), cada cámara tiene su respectivo quemador, sistema de recuperación de calor y ductos de salida de gases con respectivo sistema de filtrado y control automático de temperatura.

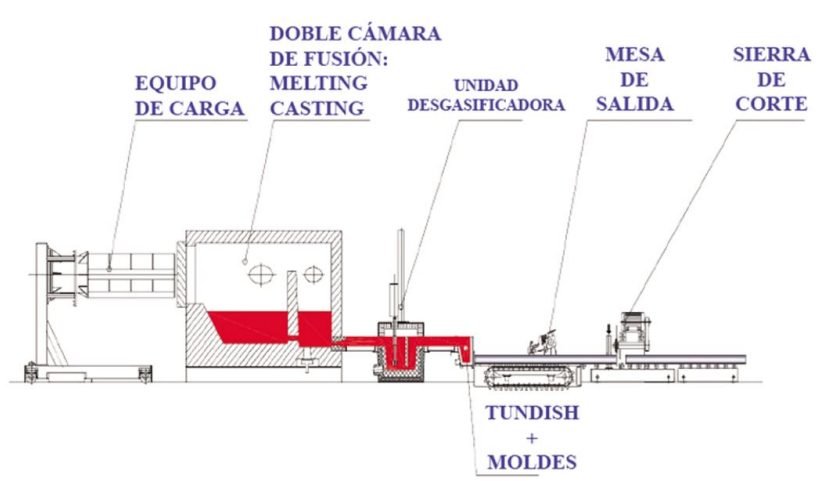

Figura 1. Equipo completo para proceso de colada continua horizontal. [1]

Equipo de casting: Contiene el sistema de control de flujo, los canales de moldeo que guían la aleación a la unidad desgasificadora que posee su rotor con impulsor de grafito para inyectar argón y refinador de grano.

Tundish: En conjunto con el filtro se encarga de albergar el aluminio para suministrar a los moldes y permitir la producción continua de billets.

Moldes: Se encuentran adaptados en la base de tundish, son elaborados con aleación de cobre; poseen forma circular con sistema de lubricación combinado con aceite biodegradable y grafitos, tiene un sistema de refrigeración con agua.

Mesa de moldeo horizontal: También llamada mesa de salida donde facilita el avance guiado de los billets moldeados con un sistema de rodillos y cadena. Sierra de corte: Conocida también como sierra volante, corta automáticamente los billets según longitud especifica de acuerdo con el lote de producción.

\section{Materiales y métodos}

\subsection{Toma de muestras y procedimientos}

El presente análisis cuantitativo de los elementos aleantes principales precipitados, en su inicio no cuenta con datos históricos de estudios anteriores ni métodos experimentales de estudio aplicados en proceso de colada continua horizontal en billets de aluminio AA6063.

En la comparación de muestras entre casting y tundish se decidió tomar 22 muestras respectivamente porque control de calidad ase dos muestras de tundish en cada hora por turno de ocho horas de producción y someter las muestras a ensayo de espectrometría para controlar los niveles de aleación basados en norma ASTM E716 y E1251 con discos tipo B.

La recolección de datos estadísticos descriptivos sobre la relación entre temperatura de moldeo y el avance de mesa, fue realizada en los turnos de trabajo durante la producción de la aleación A y B, mismas que serán descritas en el literal 2.2 y se los obtuvieron por medio de tableros de control y medición de avance de mesa con cronómetro en intervalos de tiempo cada 10 minutos.

Para ensayos de dureza Brinell y espectrometría de los billets, fueron necesarios un total de ocho billets (cuatro de aleación A y cuatro de aleación B) con un costo de producción de $\$ 700$ USD cada uno, luego se compararon rangos de aleación con el objetivo de discriminar información y quedarse con un billet por cada aleación con la intención de realizar cortes consecutivos con espesor promedio de $35 \mathrm{~mm}$ y reservándose un corte mayor de la sección media a fin de obtener las probetas para ensayo de tracción, medición de dureza y espectrometría completando así un total de 59 muestras por billet.

\subsection{Comparación entre muestras de casting y tundish}

Esta comparación espectrométrica sirve para averiguar si existe una diferencia significativa en los niveles de aleantes principales al momento de tomar una muestra de casting o tundish.

Se debe tener en cuenta lo siguiente: todos los valores a ser mencionados durante todo este análisis corresponden al porcentaje en peso de los aleantes principales (magnesio y silicio) en aleación 6063 .

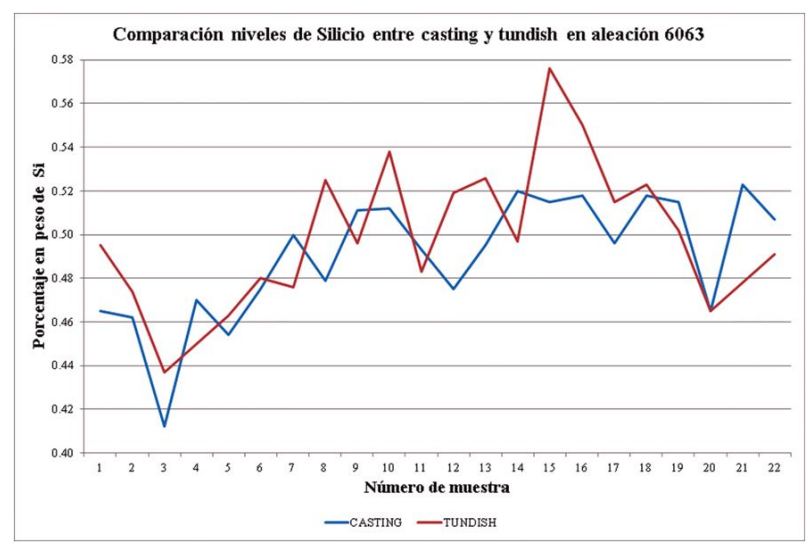

Figura 2. Comparación niveles de Si entre casting y tundish.

$\mathrm{Al}$ realizar los ensayos espectrométricos para conocer los porcentajes en peso de silicio (Figura 2), en ambos casos se obtuvo que un cincuenta por ciento 
Navas et al. / Análisis cuantitativo de los elementos aleantes principales precipitados en billets de aluminio de aleación 6063 en equipo de fundición de colada continua horizontal para el proceso de extrusión

de las muestras están por encima del valor 0,496 con un valor máximo en casting de 0,523 y en tundish de 0,576 , el otro cincuenta por ciento en ambos casos está por debajo del valor 0,496 con un valor mínimo en casting de 0,412 y en tundish de 0,437.

En promedio los niveles de silicio de la aleación se encuentran en 0,49 en casting y desviándose 0,028 unidades del promedio. De la misma forma, el nivel promedio de silicio en la aleación tomada de tundish es de 0,498 con una desviación estándar de 0,034 unidades.

Continuando, en la Figura 3 se observa la respectiva comparación entre casting y tundish en relación con el porcentaje en peso de magnesio, por medio de los ensayos espectrométricos se obtuvo que en casting un cincuenta por ciento de las muestras están por encima del valor 0,508 con un valor máximo de 0,559 y el otro cincuenta por ciento está por debajo del valor 0,508 con un valor mínimo de 0,439. En el caso de las muestras tomadas de tundish, el cincuenta por ciento está por encima del valor 0,528 con un valor máximo de 0,591 ; el porcentaje restante está por debajo del valor de 0,528 con un valor mínimo de 0,461 .

En promedio los niveles de magnesio de la aleación se encuentran en 0,508 en casting con una desviación estándar de 0,035 unidades. De la misma forma, el nivel promedio de magnesio en la aleación tomada de tundish es de 0,524 y desviándose 0,035 unidades del promedio.

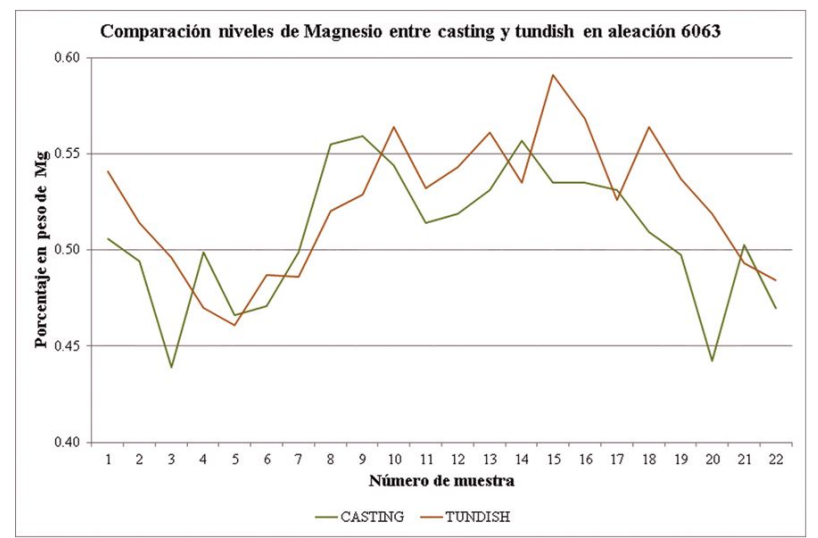

Figura 3. Comparación niveles de $\mathrm{Mg}$ entre casting y tundish.

Considerando la diferencia encontrada al momento de tomar muestras en casting y tundish, indistintamente del tipo de aleación a ser producida es preferible tomar muestras de tundish cuando se precisa equiparar los niveles de aleantes principales.

De aquí en adelante se va a denominar la aleación con niveles normales como aleación «A» y los niveles de porcentaje en peso a controlar se muestran en la Tabla 1.
Tabla 1. Rango de porcentaje en peso de elementos principales a controlar durante proceso de colada continua horizontal en aleación "A".

\begin{tabular}{ccc}
\hline \multicolumn{3}{c}{ Rango de porcentaje en peso de } \\
elementos principales en aleación «A & $\mathbf{A}$ " \\
\hline Elemento & Máximo & Mínimo \\
\hline Magnesio $(\mathrm{Mg})$ & 0,5 & 0,48 \\
Silicio $(\mathrm{Si})$ & 0,49 & 0,44 \\
Hierro $(\mathrm{Fe})$ & 0,2 & $\begin{array}{c}\text { No aplica } \\
\text { según norma }\end{array}$ \\
\hline
\end{tabular}

En cambio, de aquí en adelante se va a denominar la aleación con niveles experimentales como aleación «B», cuyos rangos de porcentaje en peso de los elementos principales a controlar se indican en la Tabla 2.

Tabla 2. Rango de porcentaje en peso de elementos principales a controlar durante proceso de colada continua horizontal en aleación "B".

\begin{tabular}{ccc}
\hline \multicolumn{3}{c}{ Rango de porcentaje en peso de } \\
elementos principales en & aleación $« \mathbf{B} »$ \\
\hline Elemento & Máximo & Mínimo \\
\hline Magnesio $(\mathrm{Mg})$ & 0,49 & 0,45 \\
Silicio $(\mathrm{Si})$ & 0,42 & 0,38 \\
Hierro $(\mathrm{Fe})$ & 0,17 & $\begin{array}{c}\text { No aplica } \\
\text { según normas }\end{array}$ \\
\hline
\end{tabular}

\subsection{Relación entre temperatura de moldeo y velocidad de avance de mesa}

La relación con respecto a dos variables importantes como son la temperatura registrada de moldeo en tundish y la velocidad de avance de mesa, se determinó que la velocidad de avance de mesa está en relación directa a la temperatura en tundish.

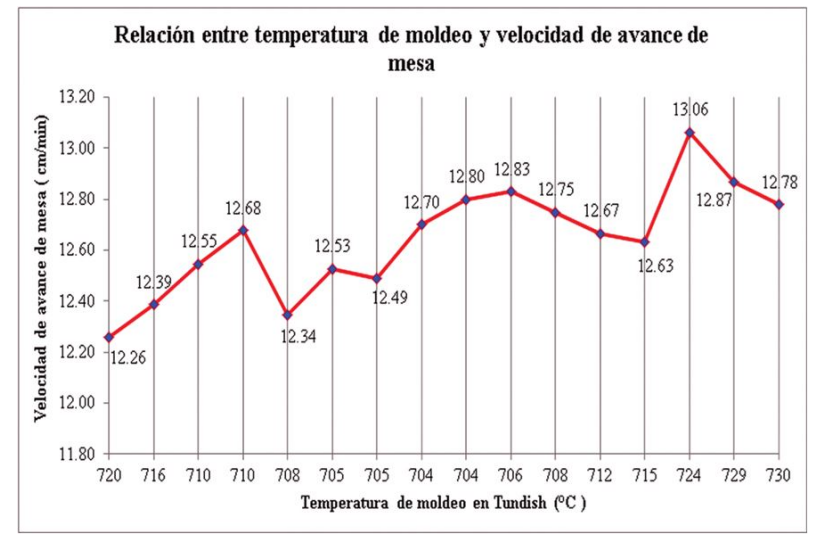

Figura 4. Velocidad avance de mesa vs. temperatura de moldeo. 
El control del avance de mesa es automático y es fácil apreciar en ciertos puntos de la Figura 4 que el mismo sistema trata de regular esa velocidad en relación con la temperatura de moldeo en tundish.

\subsection{Ensayos de dureza Brinell}

El ensayo respectivo de dureza Brinell (HB) tuvo lugar en el laboratorio de calidad perteneciente a la empresa CEDAL S. A., mismo que se lo realizó bajo norma ASTM-E10. Con una fuerza aplicada de $613 \mathrm{~N}$ con el identador de bola de diámetro 2,5 $\mathrm{mm}$. Los resultados obtenidos se los resume en la Tabla 3 .

Tabla 3. Rango de porcentaje en peso de elementos principales a controlar durante proceso de colada continua horizontal en aleación "B".

\begin{tabular}{|c|c|c|}
\hline \multicolumn{3}{|c|}{ ENSAYO DE DUREZA BRINELL } \\
\hline Departamento: & \multicolumn{2}{|c|}{ Fundición } \\
\hline Origen: & \multicolumn{2}{|c|}{ As cast } \\
\hline Proceso: & \multicolumn{2}{|c|}{ Colada continua } \\
\hline Aleación: & \multicolumn{2}{|c|}{6063} \\
\hline Longitud de billet: & \multicolumn{2}{|c|}{$2794 \mathrm{~mm}$} \\
\hline Tratamiento térmico: & \multicolumn{2}{|c|}{ Homogenizado } \\
\hline Total muestras: & \multicolumn{2}{|c|}{59} \\
\hline $\begin{array}{l}\text { Mediciones por } \\
\text { muestra: }\end{array}$ & \multicolumn{2}{|c|}{7} \\
\hline Identador & \multicolumn{2}{|c|}{$2,5 \mathrm{~mm}$} \\
\hline Fuerza: & \multicolumn{2}{|c|}{$613 \mathrm{~N}$} \\
\hline Tiempo: & \multicolumn{2}{|c|}{$10 \mathrm{~s}$} \\
\hline Tipo de aleación: & A & B \\
\hline Promedio & 47,6 & 47,34 \\
\hline Mediana & 46,08 & 46,65 \\
\hline Desviación estándar & 3,6 & 3,33 \\
\hline Máximo & 56,5 & 57,31 \\
\hline Mínimo & 43,08 & 42,7 \\
\hline
\end{tabular}

Los valores obtenidos referentes a nivel de dureza Brinell, en el billet de aleación «A» se obtuvo que un cincuenta por ciento del billet posee niveles de dureza que están sobre el 46,08 HB alcanzando una dureza máxima de 56,0 HB y el otro cincuenta por ciento del billet posee niveles de dureza por debajo de 46,08 HB llegando a una dureza mínima de 43,08 HB y con una dureza promedio en su total de 47,60 HB. La distribución de los niveles de dureza HB transversalmente en los siete puntos de medición del billet de aleación «A se contempla en la Figura 5.

La aleación «B» en todo el billet, un cincuenta por ciento posee niveles de dureza sobre los 46,65 $\mathrm{HB}$ con un máximo de $57,31 \mathrm{HB}$ y el otro cincuenta por ciento del mismo alcanzó niveles por debajo del 46,65 HB con un mínimo de 42,70 y alcanzando una dureza promedio en todo el billet de 47,34 HB. A continuación se puede avistar los niveles de dureza HB transversal de la aleación «B» en la Figura 6.

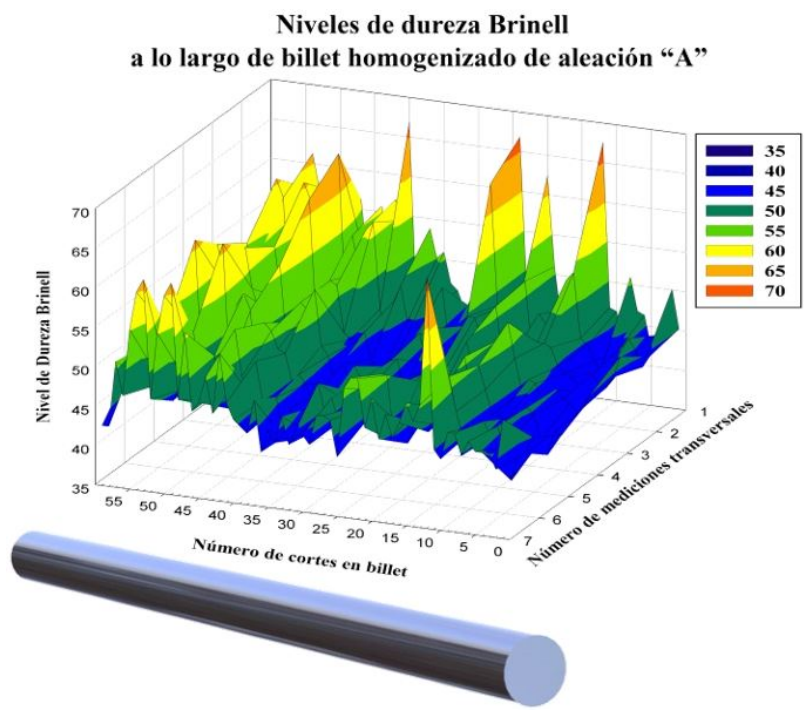

Figura 5. Dureza Brinell a lo largo del billet homogenizado de aleación «A».

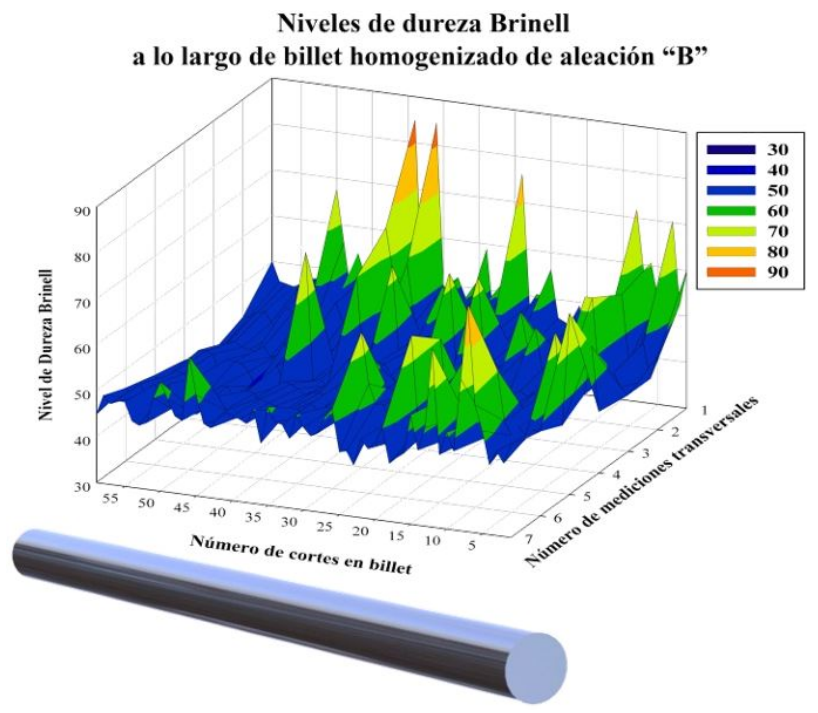

Figura 6. Dureza Brinell a lo largo del billet homogenizado de aleación «B».

Gráficamente advierte en la Figura 7 la comparación entre ambas aleaciones. Al contrario de lo que se podría esperar por los niveles en aleantes principales que posee la aleación «B», esta posee apenas en promedio un $0,55 \%$ menor dureza que la aleación «A».

\subsection{Cuantificación de los aleantes principales precipitados}

La cuantificación de los elementos aleantes principales precipitados en la aleación 6063, parte al comprender el concepto que el magnesio y silicio precipitan 
para formar siliciuro de magnesio $\left(2 \mathrm{Mg}+\mathrm{Si}=\mathrm{Mg}_{2} \mathrm{Si}\right)$, mismo que fue descrito anteriormente.

El cálculo es posible al poder obtener por medio de espectrometría el porcentaje en peso de silicio y magnesio que son los principales elementos aleantes en aleaciones de la serie 6000 .

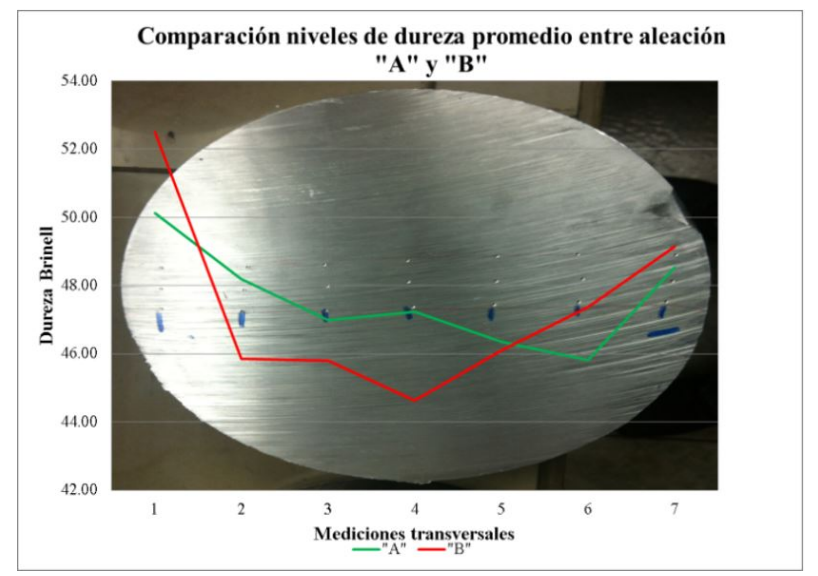

Figura 7. Comparación dureza transversal en billets homogenizados de aleaciones $« \mathrm{~A} » \mathrm{y} \ll \mathrm{B} »$.

La cuantificación, se realizó aplicando la relación estequiométrica entre magnesio y silicio por encontrarse en la formulación química del $\mathrm{Mg}_{2} \mathrm{Si}$ porque al ser un cuerpo compuesto claramente se ve que posee dos átomos de magnesio y un átomo de silicio, si se conoce el peso atómico de cada elemento se obtiene la siguiente razón para las aleaciones de la serie 6000: [3-7]

$$
\frac{M g}{S i}=\frac{48,61}{28,0855}=1,73
$$

Si la relación indica niveles mayores a 1,73, la aleación posee exceso de $\mathrm{Mg}$, menor a 1,73 la aleación posee exceso de Si y si es igual a 1,73 la aleación es de tipo estequiométrica.

La aleación «A» con porcentaje en peso promedio: Si $(0,4969), \mathrm{Fe}(0,2081), \mathrm{Mg}(0,547), \mathrm{Mn}(0,0111) \mathrm{y}$ aplicando la ecuación 1 se obtuvo 1,101 lo que demuestra la aleación posee exceso de silicio, adicionalmente en la Figura 8 se examina gráficamente la distribución longitudinal de elementos principales de control en aleación «A» y de la misma forma en la Figura 9 lo correspondiente a la aleación «B».

Para la aleación «B» registra con porcentaje en peso promedio: Si $(0,4169)$, Fe $(0,1663), \mathrm{Mg}(0,4055)$, Mn $(0,0273)$ de la misma manera aplicando ecuación 1 se obtuvo 0,973 menor a 1,73.

En la formación de $\mathrm{Mg}_{2} \mathrm{Si}$ se valora que todo el contenido de magnesio en la aleación 6063 se precipita para combinarse con la mitad de silicio disponible y así formar $\mathrm{Mg}_{2} \mathrm{Si}$; por lo contrario, estos niveles de silicio disponible se ven afectados por la presencia de hierro y manganeso en la aleación; con el fin de cuantificar el nivel de silicio disponible se procede a aplicar la siguiente ecuación: [3-7]

$$
\text { Si disponible }=\frac{\% S i-(\% F e+\% M n)}{4}
$$

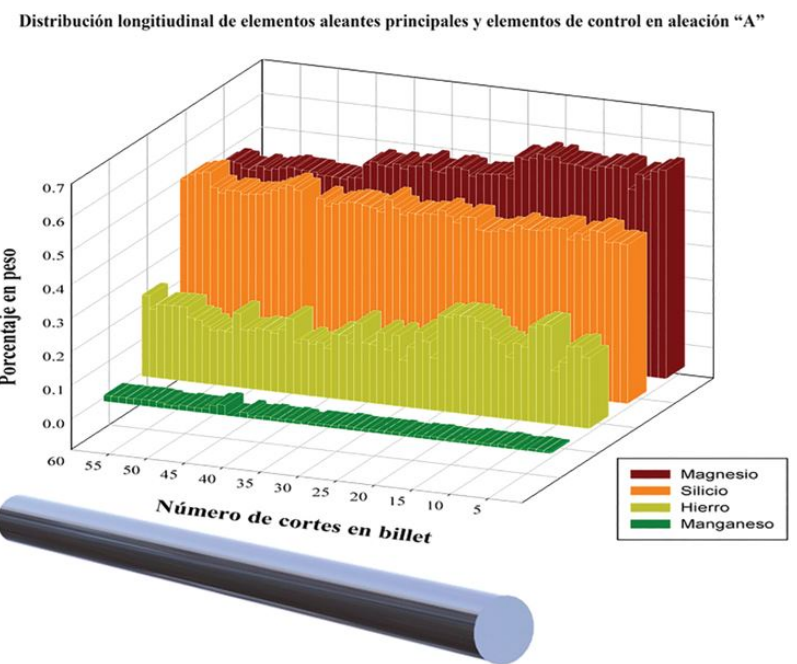

Figura 8. Distribución longitudinal en billet homogenizado de elementos principales de control en aleación « $\mathrm{A} »$.

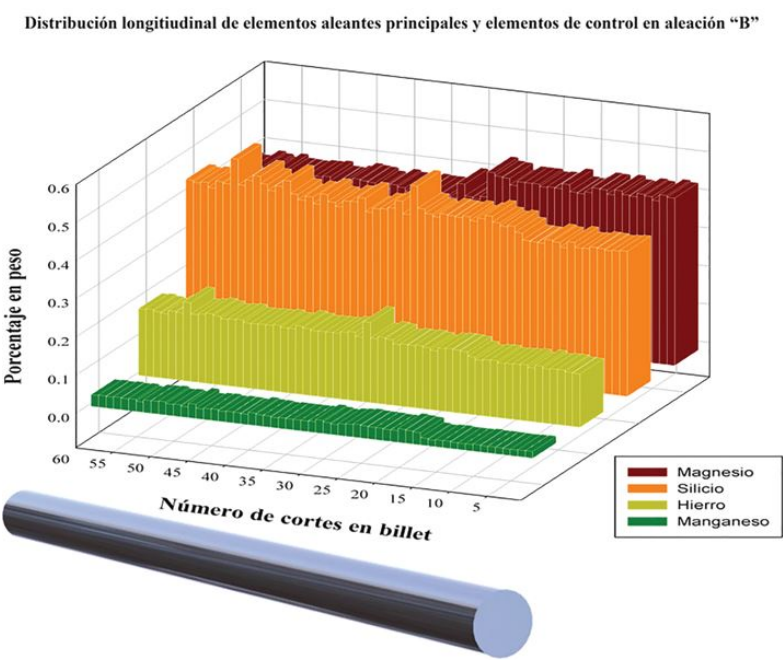

Figura 9. Distribución longitudinal en billet homogenizado de elementos principales de control en aleación «B».

Para la aleación «A» a partir de los niveles promedio obtenidos de porcentaje en peso, posee 0,442 de silicio disponible para formar $\mathrm{Mg}_{2} \mathrm{Si}$.

En el caso de la aleación «B», se obtuvo 0.369 de $\mathrm{Si}$ disponible para la formación de $\mathrm{Mg}_{2} \mathrm{Si}$.

A continuación, es necesario conocer cuánto silicio es consumido para formar $\mathrm{Mg}_{2} \mathrm{Si}$; mediante la relación estequiométrica se puede establecer aplicando la siguiente ecuación: [3-7] 


$$
\% S i=\frac{\% M g}{1,73} \cong 0,578 \times \% M g
$$

Donde los valores de Mg y Si están en porcentaje de peso de cada elemento y se obtiene para la aleación «A» un total de silicio consumido de 0,3162 para formar $\mathrm{Mg}_{2} \mathrm{Si}$.

En la aleación «B» se obtuvo 0,234 de silicio consumido para formar $\mathrm{Mg}_{2} \mathrm{Si}$.

Uno de los objetivos es el poder cuantificar los precipitados de los aleantes principales que son $\mathrm{Mg}$ y Si, sabiendo que el producto de esta precipitación es el siliciuro de magnesio. Para calcular el porcentaje de peso de $\mathrm{Mg}_{2} \mathrm{Si}$ obtenido para cada caso a partir de la siguiente ecuación: [3-7]

$$
\% M g_{2} S i=\% M g+\% \text { Si consumido }
$$

Para la aleación «A» se obtuvo un valor promedio de 0,863 de porcentaje de peso de $\mathrm{Mg}_{2} \mathrm{Si}$ y en la aleación «B» se obtuvo un valor promedio de 0,640 en porcentaje de peso de $\mathrm{Mg}_{2} \mathrm{Si}$.

Como recomendación técnica, es importante que los niveles obtenidos cuantitativamente de siliciuro de magnesio para las aleaciones de la serie 6000 debe estar entre 0,61 a 1,0 de porcentaje en peso. [2]

En promedio ambas aleaciones presentan sus niveles de siliciuro de magnesio dentro del rango recomendado técnicamente; dicha comparación es posible avistar en la Figura 10. [2]

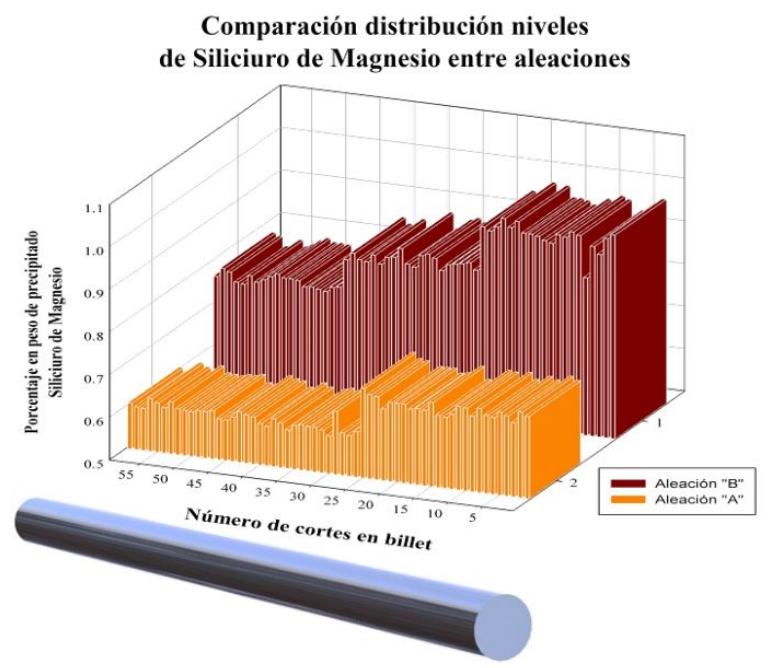

Figura 10. Comparación distribución longitudinal de siliciuro de magnesio en billet homogenizado de aleación «A» $\mathrm{y} \ll \mathrm{B} »$.

\subsection{Relación entre dureza Brinell y precipita- dos de siliciuro de magnesio}

Acerca de la relación entre dureza Brinell y porcentaje en peso de precipitados de siliciuro de magnesio en el billet de aleación «A», se puede distinguir en la Figura 11 que en secciones del billet, la dureza es inversamente proporcional al contenido de porcentaje en peso de siliciuro de magnesio, en otras palabras, a menor porcentaje en peso de $\mathrm{Mg}_{2} \mathrm{Si}$ mayor dureza Brinell y a mayor porcentaje en peso de $\mathrm{Mg}_{2} \mathrm{Si}$ menor dureza Brinell.

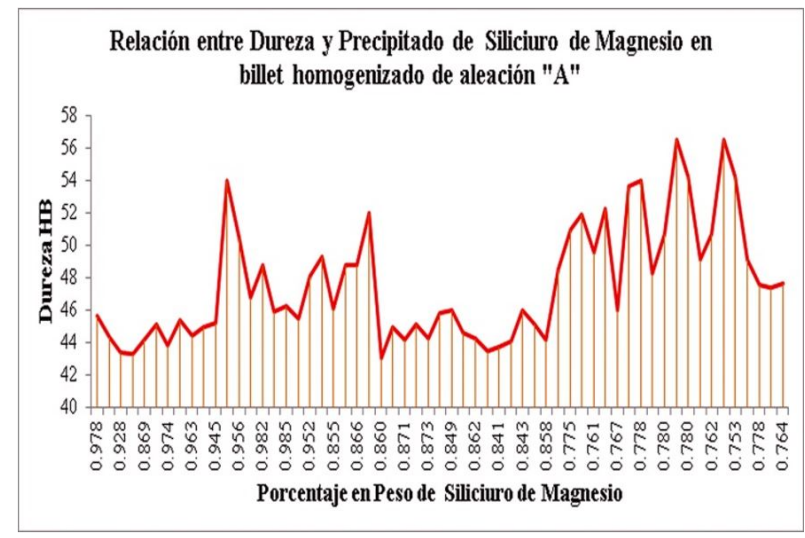

Figura 11. Relación entre dureza Brinell y precipitados de $\mathrm{Mg}_{2} \mathrm{Si}$.

Con respecto a la aleación "B", se muestra los resultados similares en la Figura 12, en secciones del billet con valores indicando una relación inversamente proporcional debido a secciones con niveles altos en porcentaje en peso de $\mathrm{Mg}_{2} \mathrm{Si}$ que registran baja dureza Brinell y en secciones del billet con niveles altos en porcentaje en peso de $\mathrm{Mg}_{2} \mathrm{Si}$ demuestra una ba dureza Brinell.

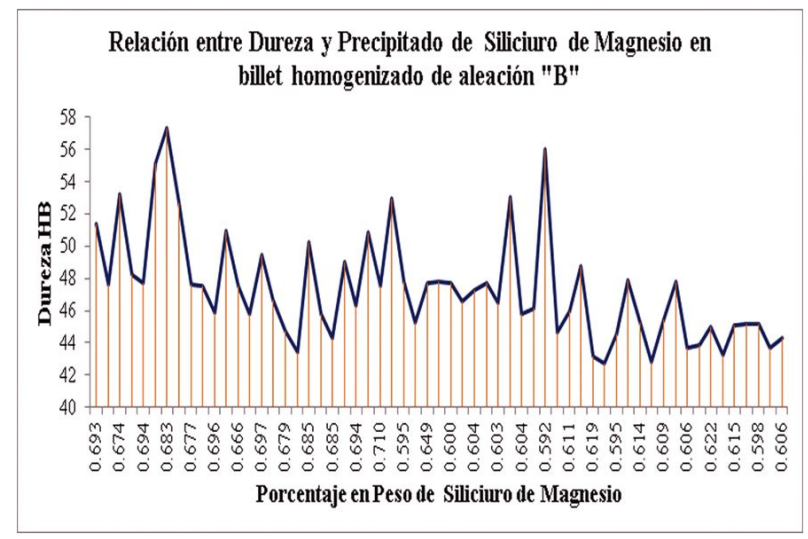

Figura 12. Relación entre dureza Brinell y precipitados de $\mathrm{Mg}_{2} \mathrm{Si}$.

\subsection{Ensayo de tracción}

No existe registro alguno de estudio in situ que haya realizado una prueba de esta naturaleza de billets homogenizados procedentes del proceso de colada continua horizontal. 
A partir de las tres probetas extraídas del billet de aleación «A», se registró un límite de fluencia promedio de $66,73 \mathrm{MPa}$ y una resistencia a la tracción promedio de $153 \mathrm{MPa}$.

Tabla 4. Resultados prueba de tracción de aleación «A».

\begin{tabular}{|c|c|c|c|c|c|}
\hline \multicolumn{6}{|c|}{ RESULTADOS ENSAYOS DE TRACCIÓN } \\
\hline Departamento: & Fundición & Origen: & As cast & Aleación: & 6063 \\
\hline Proceso: & & \multirow{2}{*}{\multicolumn{4}{|c|}{$\begin{array}{r}\text { Colada continua horizontal } \begin{array}{c}\text { Tipo: } \\
\text { Homogeneizado }\end{array}\end{array}$}} \\
\hline \multirow{2}{*}{\multicolumn{2}{|c|}{$\begin{array}{l}\text { Tratamiento Térmico: } \\
\text { Probetas bajo norma: }\end{array}$}} & & & & \\
\hline & & \multirow{2}{*}{$\begin{array}{l}\text { ASTM B557M } \\
\text { Carga } \\
\text { máxima } \\
\text { registrada }\end{array}$} & \multicolumn{2}{|c|}{ Probetas analizadas: } & 3 \\
\hline Descripción & $\begin{array}{l}\text { Resistencia } \\
\text { a la tracción }\end{array}$ & & $\begin{array}{l}\text { Límite de } \\
\text { fluencia }\end{array}$ & $\begin{array}{l}\text { Carga de } \\
\text { fluencia }\end{array}$ & $\begin{array}{l}\text { Elongación } \\
\text { en } 50 \mathrm{~mm}\end{array}$ \\
\hline & $\mathrm{MPa}$ & $\mathrm{kg}$ & $\mathrm{MPa}$ & $\mathrm{kg}$ & $\%$ \\
\hline A1 & 153 & 2450 & 67,00 & 1070 & 36,30 \\
\hline A2 & 155 & 2470 & 67,40 & 1070 & 36,50 \\
\hline A3 & 151 & 2400 & 65,80 & 1050 & 34,90 \\
\hline Promedio & 153 & 2440 & 66,73 & 1063,33 & 35,90 \\
\hline
\end{tabular}

Con relación a las tres probetas procedentes del billet de aleación «B», tuvieron un límite de fluencia promedio de 52,70 MPa y su resistencia a la tracción promedio de $134 \mathrm{MPa}$.

Tabla 5. Resultados prueba de tracción de aleación «B».

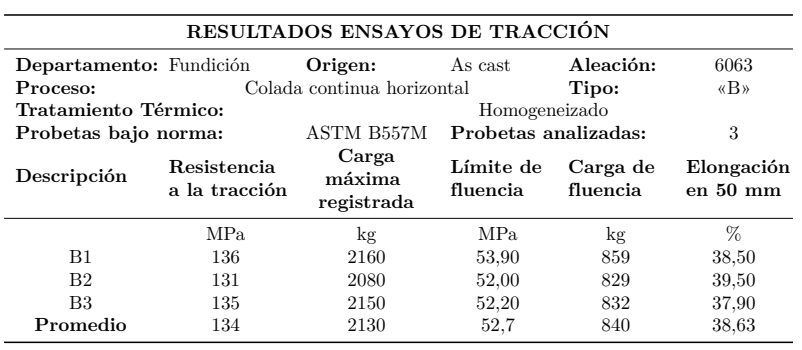

\section{Resultados y discusión}

Como resultado obtenido, se considera que la diferencia promedio entre casting y tundish es del 1,6\% con respecto a los niveles de silicio en aleación y la diferencia promedio con respecto a los niveles de magnesio en aleación es del 3,05\% debido a que varios elementos y combinaciones van a precipitar fuera de la aleación en función del descenso de temperatura; en este caso la temperatura en tundish es menor a la temperatura de casting en un promedio de $35{ }^{\circ} \mathrm{C}$. [2]

El porcentaje en peso promedio de siliciuro de magnesio en un billet de aluminio homogenizado de aleación «A» fue de 0,863 y en el billet de aluminio homogenizado de aleación «B» registró 0,64 equivalente a un $25 \%$ menos contenido de $\mathrm{Mg}_{2} \mathrm{Si}$; conforme a estos resultados no se puede contrastar con otras investigaciones porque en principio el equipo de colada continua horizontal fue diseñado para aplicar un proceso de remelting en empresas extrusoras y en el presente estudio la materia prima está compuesta por aluminio primario, perfiles extruidos y aleaciones de aluminio reciclado; por esta razón se aprecia en la Figura 8 los resultados de variación con respecto al $\mathrm{Mg}_{2} \mathrm{Si}$ a lo largo del billet debido a que el proceso es incesante y la carga de material es intermitente durante la producción, obligando a controlar los niveles de aleación de forma continua para mantener los rangos máximos y mínimos de los elementos aleantes principales.

Esta diferencia del $25 \%$ entre ambas aleaciones significó que la aleación «A» obtenga un límite de fluencia promedio de 66,73 $\mathrm{MPa}$ mayor al $21 \%$ con respecto a la aleación «B» que alcanzó un límite de fluencia promedio de 52,70 MPa; sin embargo, no existió una diferencia significativa con respecto a su dureza en escala Brinell, porque la aleación «A» es apenas 0,55\% más dura que la aleación « $\mathrm{B} » 175$ de acuerdo con los resultados expuestos en la Tabla 3.

$\mathrm{Al}$ cuantificar los niveles de precipitados de $\mathrm{Mg}_{2} \mathrm{Si}$ a lo largo del billet homogenizado tanto en la aleación «A» como en la aleación «B», y la medición de dureza Brinell, mostraron la existencia de una relación inversamente proporcional entre ambos; es decir, en puntos donde se registró un mayor porcentaje de peso promedio de $\mathrm{Mg}_{2} \mathrm{Si}$ demostró un menor nivel de dureza $\mathrm{HB}$ y en lugares con menor porcentaje de peso de $\mathrm{Mg}_{2} \mathrm{Si}$ se registró un mayor nivel de dureza $\mathrm{HB}$.

\section{Conclusiones}

A lo largo de la investigación se logró mostrar la diferencia al tomar muestras de casting y tundish; indistintamente del tipo de aleación a ser producida es preferible tomar muestras de tundish cuando se precisa equiparar los niveles de aleantes principales.

A mayor temperatura de moldeo la velocidad de avance de mesa es menor y a menor temperatura de moldeo la velocidad de avance de mesa es mayor, lo que permite determinar la existencia de una relación directa entre temperatura en tundish y velocidad de avance de mesa.

La distribución longitudinal de los niveles de $\mathrm{Mg}$ y Si en los billets AA6063 después del proceso de homogenizado, demostró la fluctuación de sus niveles a lo largo del proceso, revelando zonas de fluctuación en los valores de la aleación; otras zonas donde la aleación comienza a estabilizar sus niveles y luego otra vez muestra zonas donde la aleación presenta puntos de variación permitiendo inferir que su fluctuación a lo largo del billet son propios del proceso de colada continua horizontal.

La relación entre los niveles de precipitación de $\mathrm{Mg}_{2} \mathrm{Si}$ y el límite de resistencia a la tracción en el billet de aleación normal con un porcentaje de peso promedio de 0,863 en $\mathrm{Mg}_{2} \mathrm{Si}$, registró un valor de 153 $\mathrm{MPa}$; con respecto al billet con niveles de aleación experimental que mostró un valor promedio de 0,640 en porcentaje de peso de $\mathrm{Mg}_{2} \mathrm{Si}$ alcanzó un valor de $134 \mathrm{MPa}$ como límite de resistencia a la tracción; ultimando que existe una relación directa entre los niveles 
de precipitados de $\mathrm{Mg}_{2} \mathrm{Si}$ con respecto al límite de fluencia y resistencia a la tracción.

La cuantificación de los elementos aleantes principales, permitieron mostrar la distribución longitudinal de estos a lo largo del billet de aluminio de aleación 6063 homogenizado, mostrando una buena distribución a pesar de su naturaleza propia como proceso de colada continua horizontal y determina que este equipo a pesar de ser diseñado por HERTWICH para un proceso normal de remelting, es posible obtener billets de calidad utilizando chatarra de aluminio reciclada.

\section{Agradecimientos}

El presente estudio fue gracias a la colaboración de la empresa CEDAL S. A. en la ciudad de Latacunga, al facilitar sus instalaciones, materiales y la cooperación de su talento humano perteneciente a los departamentos de Fundición, Calidad, Matricería y Mantenimiento.

\section{Referencias}

[1] HERTWICH ENGINEERING SMS GROUP. (2016) The universal caster. [Online]. Available: www.hertwich.com

[2] M.J.G. (Jan. 2007) M.J.G'S technical recommendation, private communication.
[3] P. Barrand, R. Gadeau, and A. Dumas, "Metalurgia estructural del aluminio," Enciclopedia del Aluminio, vol. 2. PECHINEY GROUP, España: Ediciones URMO, pp. 13-30, 39-57, 61-87, 89-94, 95-123, 2001.

[4] S. E. Cordes, "Aluminum extrusion technology," Ed. Ohio, United States of America: ASM International, pp. 120-147, 2000.

[5] K. Thanaporn, J. Pearce, M. Ponboon, and U. Phongsophitanan, "Quantification of precipitated phases in 6063 aluminium billet by image analysis for improvement of homogenization condition," National Metal and Materials Technology Center, Bangkok, China, 2008.

[6] X. Fang, M. Song, K. Li, and Y. Du, "Precipitation sequence of an aged al-mg-si alloy," State Key Laboratory of Powder Metallurgy, Central South University of Changsha, China, 2011.

[7] J. Arsenio Lozano and B. Suárez Peña, "Análisis cuantitativo y caracterización morfológica de la aleación 6063. diferencias microestructurales y mecánicas entre la superficie y el núcleo de barras cilíndricas de colada semicontinua," Departamento de Ciencias de los Materiales e Ingeniería Metalúrgica, Universidad de Oviedo, España, 2012. 